\title{
Deep sequencing of human papillomavirus positive loco-regionally advanced oropharyngeal squamous cell carcinomas reveals novel mutational signature
}

\author{
Christian Grønhøj ${ }^{1 \dagger}$ (D, David H. Jensen ${ }^{1 \dagger}$, Tina Agander ${ }^{2}$, Katalin Kiss², Estrid Høgdall ${ }^{3}$, Lena Specht ${ }^{4}$, \\ Frederik Otzen Bagger ${ }^{5}$ Finn Cilius Nielsen ${ }^{5}$ and Christian von Buchwald ${ }^{1 *}$
}

\begin{abstract}
Background: The genetic profile for human papilloma virus positive ( $\mathrm{HPV}+$ ) oropharyngeal squamous cell carcinomas (OPSCC) remains largely unknown. The purpose of this study was to sequence tissue material from a large cohort of locoregionally-advanced HPV+ OPSCCs.

Methods: We performed targeted deep sequencing of 395 cancer-associated genes in 114 matched tumor/normal loco-regionally advanced HPV+ OPSCCs. Mutations and copy number aberrations were determined.

Results: We identified a total of 3459 mutations with an average of 10 mutations per megabase and a median of 28 variants per sample. The most frequently mutated genes were KALRN (28\%), SPTBN1 (32\%), KMT2A (31\%), ZNRF3 (9\%), BNC2 (12\%), NOTCH2 (25\%), FGFR2 (12\%), SMAD2 (6\%), and AR (13\%). Our findings were dominated by COSMIC signature 5 and 12, represented in other head and neck cancers and in hepatocellular carcinomas, respectively.

Conclusions: We have identified multiple genetic aberrations in HPV+ OPSCCS, and the COSMIC signature 12 as most prevalent. The mutations harbour both therapeutic and prognostic potential.
\end{abstract}

Keywords: Gene sequencing, Deep sequencing, HPV, Human papilloma virus, Oropharyngeal cancer

\section{Background}

Head and neck squamous cell carcinoma (HNSCC) is among the most prevalent cancers worldwide partly due to the growing number of oropharyngeal squamous cell carcinomas (OPSCCs) associated with human papillomavirus infection $(\mathrm{HPV}+)[1,2]$. Compared with the HPV-negative OPSCCs, this subset of cancer is associated with favourable outcome likely explained by a difference in immunological [3], clinical [4, 5], and histopathological features [6]. Consequently, the interest in the mutational profile of HPV-associated HNSCCs is growing.

\footnotetext{
* Correspondence: christian.buchwald@regionh.dk

${ }^{\dagger}$ Christian Grønhøj and David H. Jensen contributed equally to this work.

${ }^{1}$ Department of Otorhinolaryngology - Head and Neck Surgery and

Audiology, Rigshospitalet, University of Copenhagen, Blegdamsvej 9, 2100

Copenhagen, Denmark

Full list of author information is available at the end of the article
}

High-throughput DNA sequencing has led to identification of alterations in genes and pathways involved in the tumorigenic processes. With the exception of two smaller studies addressing structural DNA changes and mutations in HPV+ OPSCCs [7-9], large-scale studies have primarily mapped the diverse genetic landscape of HPV negative HNSCCs [10, 11]. Unlike a number of adenocarcinomas, no targetable genetic aberrations for OPSCCs are approved for treatment or as prognostic biomarkers. OPSCC patients are generally treated according to stage; typically for advanced disease with radiation, chemotherapy, or both, and for low stage disease surgery. The anti-EGFR-antibody (e.g. cetuximab) is the only approved targeted therapy but is has shown low to moderate effect in single-drug trials, and no convincing results as predictive biomarker [12-14].

Our knowledge about the mutational profile for HPV+ OPSCCs is incomplete, but carries the prospect of

(c) The Author(s). 2018 Open Access This article is distributed under the terms of the Creative Commons Attribution 4.0 International License (http://creativecommons.org/licenses/by/4.0/), which permits unrestricted use, distribution, and reproduction in any medium, provided you give appropriate credit to the original author(s) and the source, provide a link to the Creative Commons license, and indicate if changes were made. The Creative Commons Public Domain Dedication waiver (http://creativecommons.org/publicdomain/zero/1.0/) applies to the data made available in this article, unless otherwise stated. 
identification of targets for drug intervention as well as prognostic biomarkers for patient stratification in trial design. The purpose of this study is to present a comprehensive assessment of genetic aberrations in loco-regionally advanced HPV+ OPSCCs.

\section{Methods}

We included 114 matched tumor- and normal tissues from patients diagnosed with a HPV+ OPSCC in eastern Denmark $[1,15,16]$. The patients were identified through the Danish Head and Neck Cancer group (DAHANCA) database and validated through the national Danish Pathology Data Registry (DPDR). An expert head and neck pathologist validated the diagnosis of OPSCC from a hematoxylin and eosin (H\&E-) stained section of each tumour. The p16-staining was considered positive if a strong and diffuse nuclear and cytoplasmic reaction was present in more than $75 \%$ of the tumour cells [17]. Formalin-fixed paraffin embedded (FFPE) tumour specimens was handled according to standard operating procedures for the p16 immunohistochemistry. The protocol for p16 immunohistochemistry and HPV DNA PCR is previously described in detail $[1,16]$. Smoking was quantified in number of pack-years (one pack year equals 20 cigarettes per day for one year), and data was collected from medical files. From an $H \& E$-stained section, tumor and normal tissue was contoured by a head and neck pathologist. Tumor and normal tissue was subsequently punched biopsied from the FFPE-block to avoid contamination.

\section{Sequencing data generation and analysis}

A custom panel of 395 cancer-associated genes (Additional file 1: Table S1) were targeted and sequenced in tumour-normal pairs. Genes were identified via cBioportal.org and selected based on results from The Cancer Genome Atlas [18], Agrawal et al. [19], and Rubio-Perez et al. [20]. Median coverage of the targeted bases was $150 \mathrm{X}$ in the tumours and $200 \mathrm{X}$ in the matched normal tissue.

\section{DNA extraction}

Deamination of cytosine bases to uracil is a known source of DNA damage occurring in FFPE blocks that may lead to cytosine-thymine conversion (artefacts) in PCR and/or sequencing reactions [21], that can be ameliorated with the use of an Uracil-N-Glycosylase [22]. Therefore, we used the commercial GeneRead DNA FFPE Kit (Qiagen, Hilden, Germany), which is based on specific removal of deaminated cytosine residues from FFPE DNA using the Uracil-N-Glycosylase enzyme. The kit was used with the manufacturer's instructions, except for the use of double amount of proteinase $\mathrm{K}$ and deparaffination solution, and samples were left overnight for proteinase $\mathrm{K}$ digestion at $56^{\circ} \mathrm{C}$.

\section{Library preparation and sequencing}

Genome libraries were prepared according to the Ovation Custom Target Enrichment System protocol (NuGen). Landing-probes were designed by NuGEN to target the chosen genes. Target enrichment and library preparation was done according to the manufacturer's protocol (NuGEN Technologies, San Carlos, CA). Briefly, genomic DNA was fragmented, end-repaired and ligated with forward barcoded adaptors, followed by a bead purification step. The barcoded adaptor contained both an 8-nt sample-specific barcode and a 6-nt UMI. The latter was used to identify and remove duplicate reads. The reverse adaptor was annealed to the target regions and extended. The library amplification step comprised 25 PCR cycles followed by bead purification and sequencing. Sequencing was carried out on a NextSeq 500 (Illumina Inc., San Diego, CA) with single-end sequencing (150-bp) using the high-output kit v2.

\section{Bioinformatics analysis}

Raw sequencing data (.bcl files) were demultiplexed into individual FastQ read files with Illumina's bcl2fastq v2.16.0.10 (Illumina Inc., San Diego, CA) based on their unique index, with the R1 read containing the forward read and R2 containing the UMI. Each sequencing library was checked for quality with fastQC (version 0.11.5). A combination of cutadapt (version 1.11), BBDuk (version 35.82) and ERNE-filtering (version 2.1.1) was employed to remove adapter and linker sequences, remove trailing probe sequence on R1, and clip low-quality bases ends. Read alignment was carried out with the BWA algorithm (BWA version 0.7.10) with UCSC hg19 (GRCh37) as the reference. Deduplication was performed with an in-house script developed at IGA Technology (http://igatechnology.com), which makes use of the UMI to accurately remove PCR duplicates. The deduplicated reads were subsequently prepared for variant calling after realignment to correct for misalignments around indels and recalibrating base qualities based on known polymorphisms present in the human dbSNP (dbSNP137) with the GATK tool (v3.6-0-g89b7209). Variants were called with Mutect2 based on tumour-normal pairs with standard settings. Only mutations that passed Mutect2 filters were used in the downstream analysis. We performed a filtering step before annotation, where SNPs present in the European 1000 Genomes cohort [23] with an allele frequency above $1 \%$ were removed, as they are more likely to represent common population polymorphisms than somatic mutations. We also filtered SNPs that did not full-fill the following criteria: Number of mutant allele reads in tumour sample $\geq 5$, total number of allele reads $\geq 20$, 
quality score $(\mathrm{QSS})>20$ and variant allele fraction $>0.1$, in order better to exclude $\mathrm{C}>\mathrm{T}$ variants associated with FFPE artefacts [24]. To account for false positive variants from Mutect2 samples with a SQSS > 20 was filtered, where SQSS is QSS/allelic depth in the tumour [25]. For annotation, we used Oncotator v. 1.8.1.0 [26]. For visualization of mutations we used maftools [27]. Somatic genes that were significantly mutated were identified using OncodriveFML 1.1 using a threshold of $\mathrm{q}<0.1$ with CADD v.1.3, a signature by cancer type and coding regions [28]. Mutational signatures were predicted in each sample using the COSMIC framework [29]. Substitution mutations across the whole genome were analysed in context of the flanking nucleotides (96 possible trinucleotide combinations). Identified signatures were compared with 30 other validated signatures, and the frequency of each signature per megabase was determined. The numbers of enriched mutational signatures were established by a best-fit rank based on decreasing cophenetic correlation coefficients. Apolipoprotein B mRNA editing enzyme, catalytic polypeptide-like (APOBEC) enrichment scores were estimated as described by Burns et al. [30]. Briefly, enrichment of $\mathrm{C}>\mathrm{T}$ mutations occurring within a tri-nucleotide DNA sequence $(\mathrm{tCw})$ motif over all of the $\mathrm{C}>\mathrm{T}$ mutations in a given sample was compared to background cytosine ratio and tCw's occurring within $20 \mathrm{bp}$ of mutated bases. Samples were classified as APOBEC-enriched based on an APOBEC enrichment score $>2$ and/or false discovery rate $(\mathrm{FDR})<0.05$. DNA copy number variations $(\mathrm{CNV})$ were predicted by analysing the panel-sequencing data using CNVPanelizer, which compares tumour samples with a pool of normal tissue samples. The algorithm combines bootstrapping the reference set with the subsampling of amplicons associated with each of the target genes. This serves as a non-parametric estimation of the distribution of the gene-wise mean ratio between healthy reference samples and each tumour sample. All normal samples were used as a reference for calculating the sample-wise CNVs. Only genes that passed the noise and significance filter are reported as amplifications or deletions. To compare the difference in mutations between patients with high and low exposure to tobacco, we performed a fisher test on all genes between the two conditions to detect differentially mutated genes.

\section{Results}

We sequenced 114 locoregionally-advanced HPV-positive oropharyngeal squamous cell carcinomas. The targeted sequencing from 395 genes identified a total of 3459 mutations with an average of 10 mutations per megabase (excluding silent variants). The majority of mutations were missense mutations (86\%), and less common mutations were frame-shift-deletions (5\%), splice-site mutations (5\%) and non-sense mutations (4\%) (Fig. 1). The majority of identified mutations were SNPs, but deletions and insertions (indels) were also identified (Fig. 1). Of the 114 tumours $96 \%(n=110)$ were $\mathrm{HPV}+/ \mathrm{p} 16+$ and the remaining $\mathrm{HPV}+/ \mathrm{p} 16-$.

\section{Mutation signatures}

The predominant type of SNV was found to be $\mathrm{C}>\mathrm{T}$ and $\mathrm{T}>\mathrm{C}$ (Figs. 1 and 2). The DNA substitution mutations were most commonly transitions (Fig. 2). The mutational pattern of each sample was compared against all 30 validated mutational signatures from COSMIC [29]. This was assessed by the COSMIC algorithm that takes the sequence context of each mutation into account (Fig. 3). The tumours were dominated by COSMIC signature 5 or 12 (Figs. 2 and 3). Signature 5 has previously been observed to be enriched in HNSCC. The signature is depicted in most cancers and exhibits transcriptional strand bias for $\mathrm{T}>\mathrm{C}$ substitutions at an ApTpN context [31]. Signature 12 is known to be present in a subset of liver cancers, and exhibits a strong transcriptional strand-bias for $\mathrm{T}>\mathrm{C}$ substitutions [31]. It was also investigated whether an APOBEC signature was significantly enriched in our samples. The APOBEC signature was enriched in a quarter of the samples $(23 \%)$ [30] (Additional file 2: Figure S1).

\section{Somatic driver mutations}

The OncodriveFML $(\mathrm{q}<0.05)$ tool was employed to identify genes enriched in mutations. This algorithm identified 167 significantly mutated genes (Fig. 4). Of these $A P O B$ (35\%), BIRC6 (32\%), SPTBN1 (32\%), FAT2 (32\%), KMT2A (31\%), FAT1 (30\%), BPTF (30\%), TRIO (30\%), HERC2 (28\%), and KALRN (28\%) were among the most frequently mutated genes. The most prevalent genomic alterations were located in KALRN (28\%), SPTBN1 (32\%), KMT2A (31\%), ZNRF3 (9\%), BNC2 (12\%), NOTCH2 (25\%), FGFR2 (12\%), SMAD2 (6\%), and AR (13\%).

\section{Copy-number alterations}

We determined CNAs from our sequencing panel by employing CNVPanelizer, an algorithm specifically designed to estimate copy number frequencies using targeted massive parallel sequencing data. The most frequent gene copy gain was detected for ATF68 $(n=83$; $72 \%$ of samples) and $I L 3 R A$ ( $n=81 ; 71 \%$ of samples). We found a high level of inter-patient CNA heterogeneity, but also a high intra-patient $\mathrm{CN}$ heterogeneity.

\section{Mutant-allele tumour heterogeneity scores}

In the cohort, we identified the mutant-allele tumour heterogeneity (MATH) score. The median score was 20.8 (range 8-36) (Additional file 3: Figure S2). 


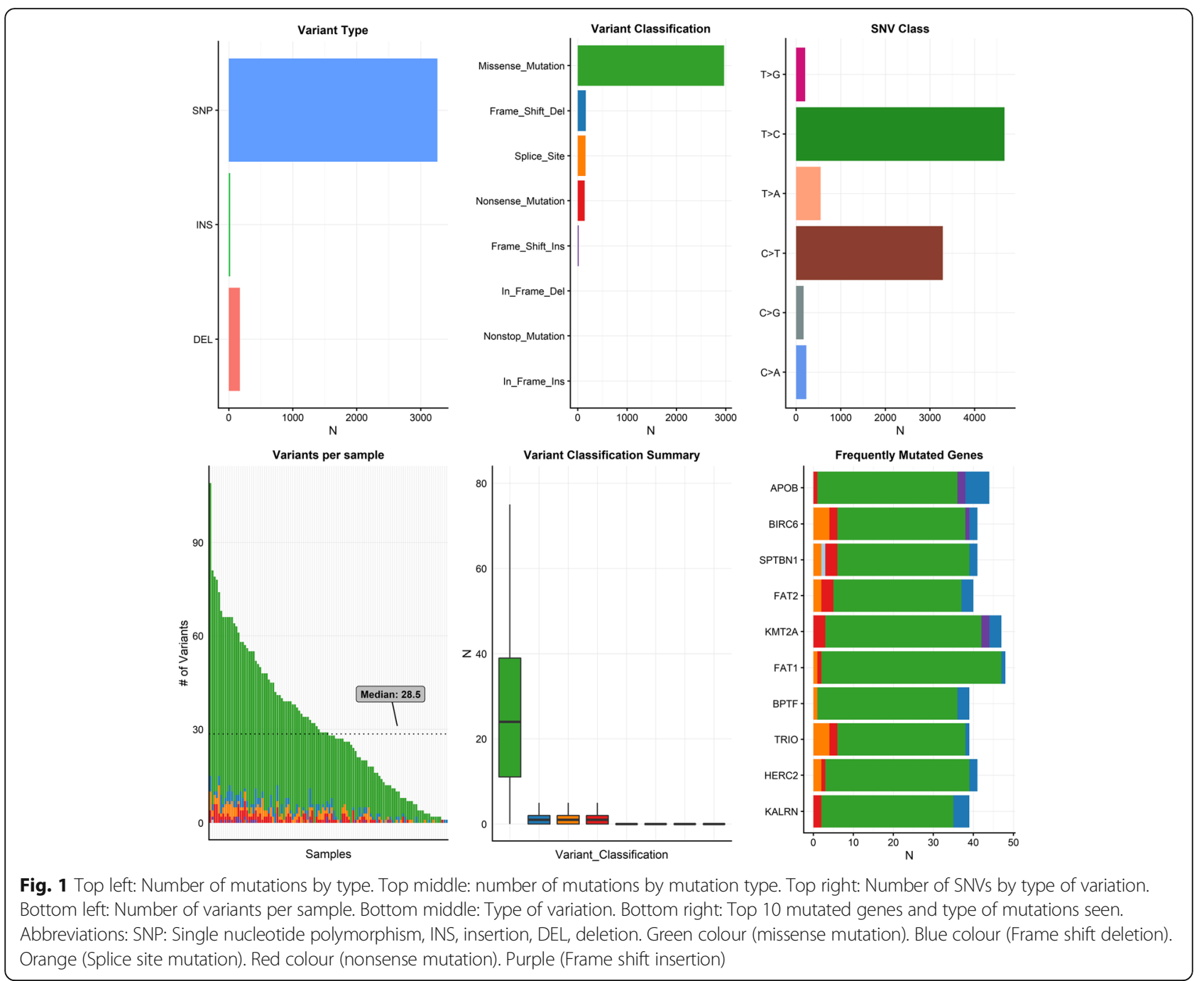

\section{Effect of smoking}

Information on smoking was available for 70 patients with a median number of pack years of 15 (95\% CL: 1.5-20 pack years). Thirty patients had never smoked or had less than 10 pack years. The genetic aberrations differed significantly between smokers and non-smokers. For smokers the genes FGFR2, EPHA2 were significantly more likely to be mutated. For non-smokers the genes IREB, BAZ2 and BRCA1 were significantly more often mutated. The mutational signature for those with low tobacco use was signature 5 and signature 12 in that order. The mutational signature for those with high tobacco use was in contrast 12 and 5. We did not observe any significant difference in MATH score between those with high and low tobacco use. There was also no significant increase in mean number of mutations per sample between these two groups.

\section{Discussion}

Here we present the targeted mutation spectrum of a large cohort of HPV-positive OPSCCs. We corroborate previously reported aberrations (e.g. FGFR, PIK3, FAT1, NOTCH2), but also more rare alterations in HPV-positive OPSCCs (e.g. KALRN, KMT2A, and SPTBN1) [11, 20, 32]. Our finding of 3459 mutations with an average of 10 mutations per megabase is higher than that in the TCGA HNSCC cohort [33], which may be explained by the fact that the TCGA cohort is predominately HPV-negative tumours.

Among human cancers, TP53 is the most frequently mutated gene, and TCGA data has shown that p53 'gain-of-functions' mutants bind to, and upregulate, several chromatin regulatory genes including the methyltransferases MLL1 - also known as KMT2A - which is highly prevalent in our cohort (31\%) [34]. A chromatin mechanism causal for the progression of tumours with gain-of-function $\mathrm{p} 53$ prospects possibilities in the design of combinatorial chromatin-based therapies [34]. Aberrations in SPTBN1, that encodes a scaffolding cellular protein involved in the formation of the cytoskeleton, is a useful prognostic biomarker in HPV-negative HNSCCs since patients with tumours expressing SPTBN1 have 


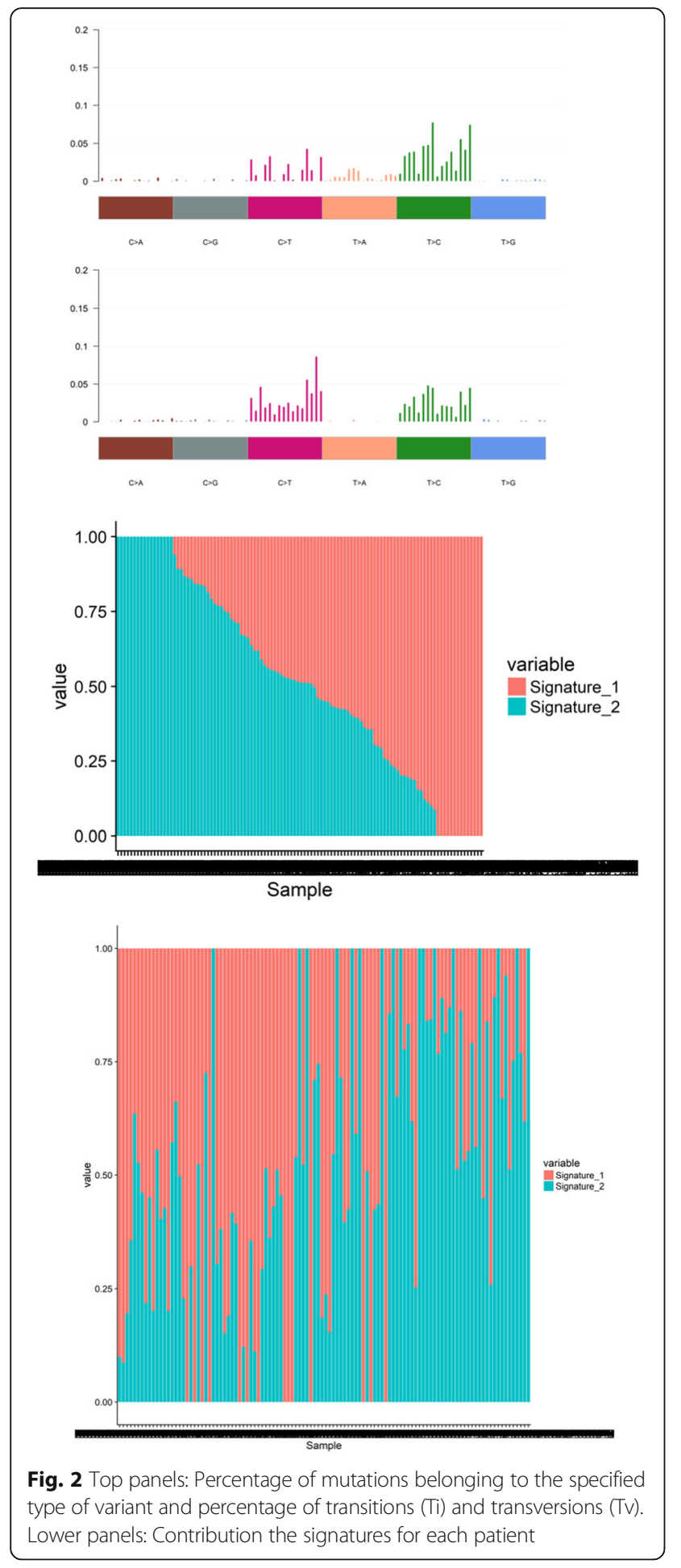

four times higher mortality, compared with patients not harbouring this mutation [35]. Although Zhu et al. examines gain-of-function, our data indicates that this gene is also prevalent in HPV+ OPSCCs and could be prognostic in these tumours as well. The known cancer gene ZNRF3 belonging to the E3 ubiquitin ligase family was also frequent in our cohort ( $9 \%$ of samples). Previously, it has been reported that ZNRF3 has the ability to inhibit the metastasis and tumorigenesis by suppressing the $\mathrm{Wnt} / \beta$-catenin signalling pathway in nasopharyngeal carcinomas (NPC), hence believed to be a potential molecular target for treatment of NPC. Based on our results, it should also be considered in HPV+ OPSCCs [36]. Due to availability of existing therapeutics and high prevalence of mutations in HPV+ OPSCC, patients with PIK3CA and CDK4/CDK6 mutations should be recommend for future phase 0 and I trials. Notably, in our cohort, we merely identified an occurrence of 7 and 1\% related to PIK3CA pathways and CCND1 aberrations. Further, the FGFR2/3 mutations are of particular interest because they were present in $20 \%$ of the tumours, in particular the S249C mutation, which is an oncogenic driven in bladder cancer [20]. Upon binding of ligand, FGFRs activate a cascade of downstream signalling pathways, such as the mitogen activated protein kinase (MAPK), phosphoinositide-3-kinase (PI3K)/Akt pathways signal transducer and activator of transcription (STAT). The mutated FGFR isoforms result in oncogenic FGFR signalling, promoting tumorigenesis, and the defect FGFR signalling pathway is a major contributing factor in the pathogenesis and progression of HNSCC [37, 38]. Inhibition of FGFRs is a promising therapeutic strategy, and phase I and II trials are progressing [39, 40] The tumor suppressor TRAF3 has previously been brought forward as a potential target for therapy development as it was inactivated in $20 \%$ of the HPV-positive tumors in the TCGA cohort. [10] Interestingly, we only identified 5\% tumors with this alteration in our cohort.

A previous study [41] has focused on mutations in HPV-positive and HPV-negative patients in a mixed population of larynx, oral cavity, oropharynx and hypopharynx cancer patients. In this study, a high prevalence of PIK3CA alterations (mutations and amplifications) was evident, which is not the case in the present study. We speculate that this could be related to 1) a high prevalence of smoking in the present study, 2) ethnical dissimilarities, and 3) the definition of being HPV-positive (RNA-sequencing versus HPV-DNA PCR). These factors could influence the carcinogenic process (smoking vs. no smoking) as well as perhaps not examining the exact same phenotype (being HPV-DNA PCR positive vs. RNA-seq positive for E6/E7), and perhaps tissue specific mutational processes (oropharynx vs. other head and neck cancer subsites).

Other studies evaluating HPV+ OPSCC consistently report mutations in PIK3CA as well as PTEN, TRAF3, NOTCH1. Although, we did identify these aberrations, they were not among the twenty most prevalent mutations in our cohort. This could be explained by the deduction that these aberrations are not as prevalent in 


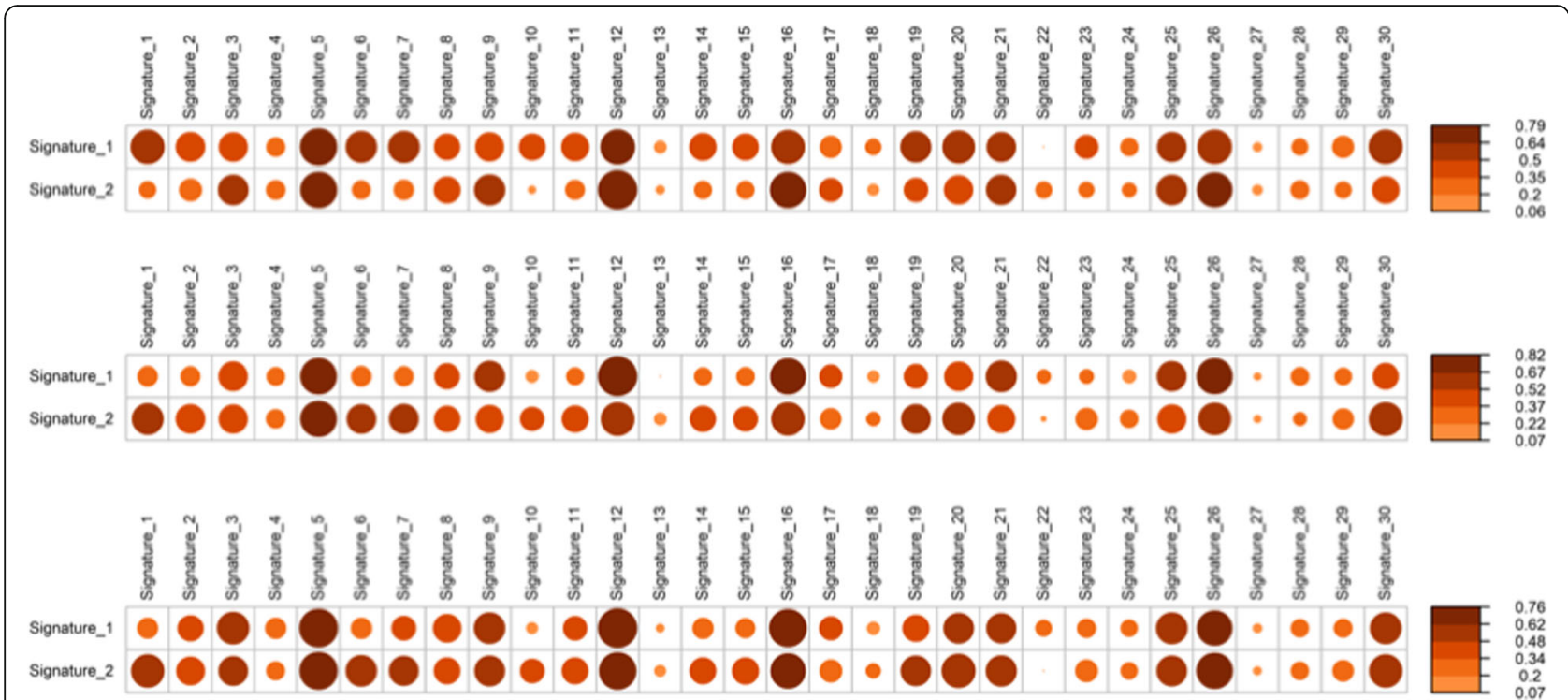

Fig. 3 Panel (Top Panel) Correlation plots for signatures for all patients. Panel (Middle Panel) Correlation plots for signatures for patients with high tobacco smoking consumption. Panel (Lower Panel) Correlation plots for signatures for patients with low tobacco smoking consumption

Denmark as other countries, or the fact that our specimens or probes were not adequate set for identifying these mutations.

Interestingly, IL3RA, HLA-A and $H L A-B$ have copy number gains in HPV positive oropharyngeal cancers [3, 42]. Absence of HLA class I (HLA-A and -B) in HPV+ OPSCC has indicated improved outcome which could be used clinically to select patients for trials with de-escalating therapy. The understanding between the genetic background of OPSCC patients and HLA-traits remains incomplete as several HLA-traits have been associated to altered outcome [43, 44].

The landscape of HPV+ and HPV- OPSCCs point to different signatures and structural alterations [11]. To stratify HPV+ from HPV- patients, the most obvious aberrations from our cohort would include KALRN, FGFR2 and NOTCH2 which are rare in HPV- HNSCC. The NFE2L2 pathway as well as the promising PIK3CA mutation and CCND1 amplication should also be prioritized.

From the TCGA cohort, the majority of driver mutations were found to be clonal (e.g. "early" mutations opposed to subclonal viewed as "late" mutations) where CDKN2A and TP53 were nearly completely clonal [45]. McGranahan et al. identified three "early" signatures in HNSCC: 1 ) $\mathrm{C}>\mathrm{T}$ transitions at $\mathrm{CpG}$ sites associated with spontaneous deamination of methylated cytosines; 2) an APOBEC-signature (also seen in "late" mutations); and 3) a smoking-associated signature. In our material, we also identify the APOBEC signature in $23 \%$ of the tumors, likewise reported in other HPV+ HNSCCs series [46].

We observed a significant increase in the COSMIC signature 12 associated with certain virus-driven liver cancers. Interestingly, a trial related to these aberrations is progressing. Ribavirin, that target the eIF4E translation initiation factor, is used to treat hepatitis $\mathrm{C}$, and is under investigation for recurrent or metastatic OPSCC (ClinicalTrials.gov, NCT02308241). Ribavirin is also being evaluated along with induction chemotherapy including afatinib (a tyrosine kinase inhibitor) and weekly carboplatin/paclitaxel for stage IV HPV-associated OPSCC (ClinicalTrials.gov, NCT01721525). Additionally for HPV + OPSCC patients with recurrent or metastatic disease, rigosertib (a PI3K (phosphatidylinositol-3 kinase) and PLK (Polo-like kinase) signalling pathway inhibitor) is being investigated in a phase II trial (ClinicalTrials.gov, NCT01807546), and in a phase I trial used as initial treatment before platinum based RT-C (ClinicalTrials.gov, NCT02107235). For high risk HPV+ OPSCC patients, the PI3K inhibitor, BYL719, is being tested with induction paclitaxel and cisplatin followed by T-site surgery, neck-disssection, and with post-operative risk adapted IMRT (ClinicalTrials.gov identifier, NCT02298595).

Although this study is strengthened by a setup, that includes a deep coverage, a high number of genes, and HPV and p16 status of all patients to ensure HPV-active infections, some limitations should be noted. First, there may be a selection bias both in patients but also because we employed a gene panel. Moreover, the particular selection of genes might influence the findings, since we have included quite large genes (e.g. APOB) where an alteration is more likely opposed to smaller genes. The sub analysis including tobacco merely includes 70 patients due to missing data and a higher number of patients might lead to other findings. Finally, as described 


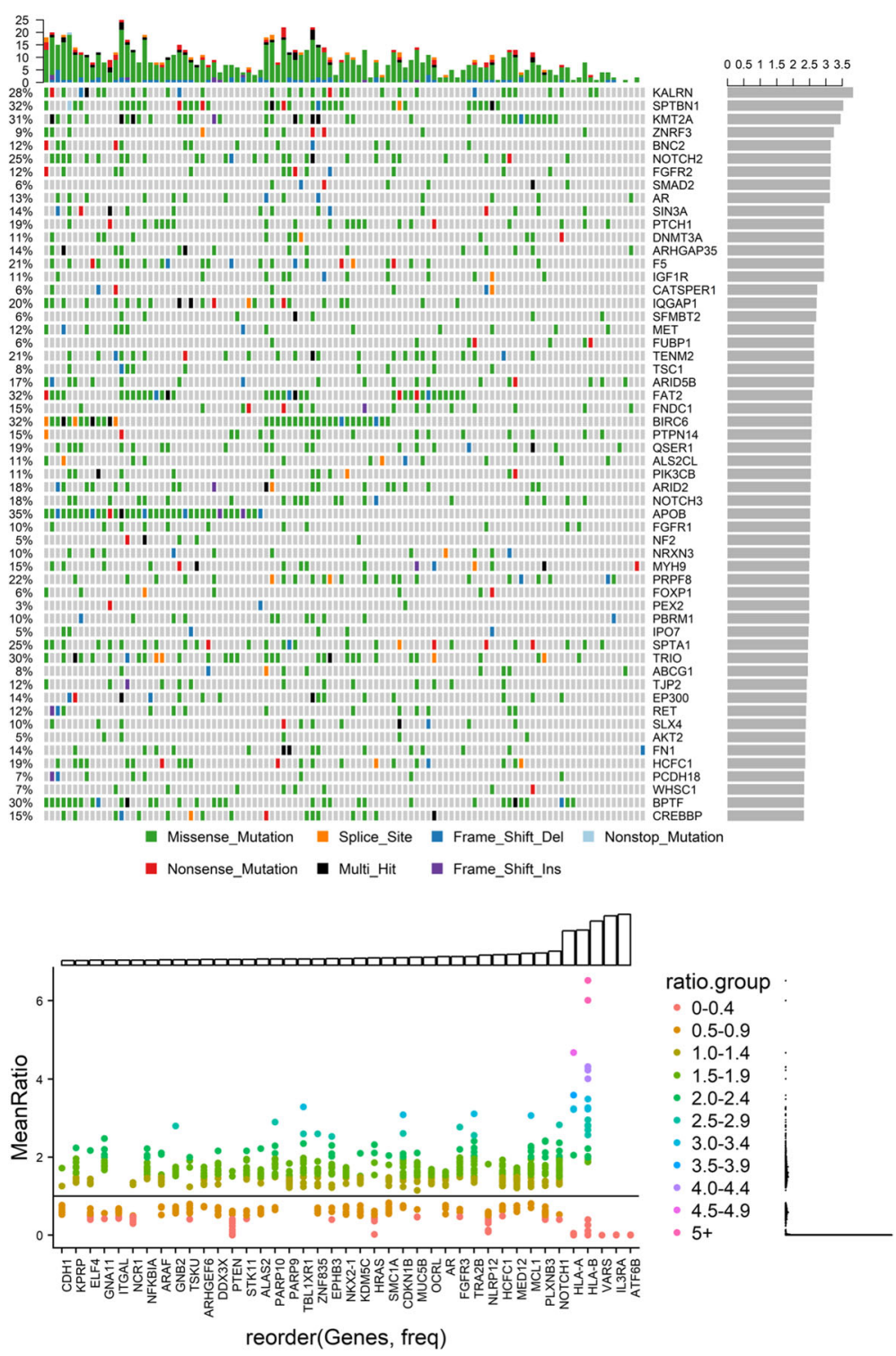

Fig. 4 Mutations in significantly mutated genes from OncodriveFML sorted by q-value. Top panel: Total coding mutations per sample. Right: -10log(q-value). Lower panel: Top Panel Type of mutation with frequency in cohort given at the left. Lower Panel The most frequent deletions and copy number alterations in the cohort from CNVpanelizer. At the top of this panel is a histogram depicting the frequency of the alteration in the entire cohort

in "Methods" section, we strained to reduce artefacts from the use of FFPE tissue (e.g. in the data analysis and tissue preparation) although it should be included as a probable source of bias. Tumor mutational burden (TMB) in the present study was defined as the median number of mutations per megabase examined in the targeted sequencing. As the present study concerns genes previously known to be implicated in carcinogenesis, the TMB would be expected to be higher than studies concerning TMB from e.g. exome-sequencing studies, as there would be fewer mutations per examined base.
When breaking down cosmic signatures from the non-smokers and smokers, we did not observe any difference in enriched cosmic mutational signatures. When looking at mutational signatures in the APOBEC enriched and non-enriched groups, the signature 5 was not enriched in the APOBEC-enriched group, but rather the signature 2. Signature 2 has been attributed to activity of the AID/APOBEC family of cytidine deaminases, and has been related to viral infections.

Both tobacco smoking and defects in DNA repair are known to induce a large number of genetic aberrations, and may be distinct ways to accumulate genetic 
aberrations required for the emergence of cancer. We found a significant difference between smokers and non-smokers underlining the importance of including tobacco-smoking consumption in prediction models and risk-stratifications. It is likely that the HPV-positive smokers acquire tobacco-related mutations but maintain virus related signatures. Even when stratified on smoking-consumption (none-smokers vs. heavy smokers), a large inter-tumor heterogeneity exists. Thus, if the future aim is to offer a personalized treatment approach it may require a very large battery of anticancer targeted drugs. Although fast-moving technologies have prompted the capability of identifying genetic aberrations promptly and precisely, it remains largely unknown which therapy(-ies) to offer based on the combinations of driver mutations. In order to fuel the development of targeted clinical trials and diagnostic testing, confirmatory studies addressing genetic aberrations in HPV+ OPSCC are needed.

\section{Conclusion}

In conclusion, $\mathrm{HPV}+\mathrm{OPSCC}$ harbour multiple genetic aberrations with both therapeutic and prognostic potential. The discovery of signatures and shared mutations from across organs (i.e. liver, lung and esophagus SCCs) might speed the progress of phase II and III trials, and should be incorporated in drug testing, especially for the heavy smokers.

\section{Additional files}

\section{Additional file 1: Table S1. 395 targeted genes. Table of targeted genes. (DOCX $26 \mathrm{~kb}$ ) \\ Additional file 2: Figure S1. APOBEC mutations. No sample was demonstrated to be significantly enriched for APOBEC mutations, and only a small fraction of mutations in each sample was of the APOBEC type. (JPG $198 \mathrm{~kb}$ )}

Additional file 3: Figure S2. MATH scores. Example of the mutant-allele tumor heterogeneity (MATH) scores, as a measure of tumor heterogeneity. The higher the math-score the higher the tumor heterogeneity. Mid right: Histogram of the MATH-score in the entire cohort. (JPG $201 \mathrm{~kb}$ )

\begin{abstract}
Abbreviations
APOBEC: Apolipoprotein B mRNA editing enzyme, catalytic polypeptide-like; DAHANCA: Danish Head and Neck Cancer group; DPDR: Danish Pathology Data Registry; FDR: False discovery rate; FFPE: Formalin-fixed paraffin embedded; H\&E: Hematoxylin and eosin; HNSCC: Head and neck squamous cell carcinoma; HPV: Human papilloma virus; MAPK: Mitogen activated protein kinase; MATH: Mutant-allele tumor heterogeneity; NPC: Nasopharyngeal carcinomas; OPSCC: Oropharyngeal squamous cell carcinomas; PI3K: Phosphoinositide-3-kinase; STAT: Signal transducer and activator of transcription; tCw: Tri-nucleotide DNA sequence; TMB: Tumor mutational burden
\end{abstract}

\section{Funding}

This study is funded by the non-profit organizations Candys Foundation and Kræeftfonden (no grant numbers). The funder had no role in the experimental design, analysis, or manuscript preparation or submission. The funder provided funds to initiate and complete the study, including investigator salaries, equipment costs, and research and clinical costs. All authors had complete access to the data by request. All authors authorized submission of the manuscript.

\section{Availability of data and materials}

The datasets used and/or analysed during the current study are available from the corresponding author on reasonable request.

\section{Authors' contributions}

CG: Conceived of the presented idea, design the study and drafted the first version of the manuscript. DJ: Conceived of the presented idea, design the study and performed the analytic calculations. Approved the final manuscript. TA: contoured tumor and normal tissue. Commented and approved the final manuscript. KK: Contoured tumor and normal tissue. Commented and approved the final manuscript. EH: Commented and approved the final manuscript. LS: Commented and approved the final manuscript. FOB: Commented and approved the final manuscript. FCN: Commented and approved the final manuscript. CVB: Conceived of the presented idea, design the study and commented and approved the final manuscript. All authors read and approved the final manuscript.

\section{Ethics approval and consent to participate}

Ethical approval is granted from the Capital Region of Denmark's regiona ethics committee (ID: H-15005784). Consent to participate was waived by Capital Region of Denmark's regional ethics committee (ID: H-15005784).

\section{Competing interests}

The authors declare that they have no competing interests.

\section{Publisher's Note}

Springer Nature remains neutral with regard to jurisdictional claims in published maps and institutional affiliations.

\section{Author details}

'Department of Otorhinolaryngology - Head and Neck Surgery and Audiology, Rigshospitalet, University of Copenhagen, Blegdamsvej 9, 2100 Copenhagen, Denmark. ${ }^{2}$ Department of Pathology, Rigshospitalet, University of Copenhagen, Blegdamsvej 9, 2100 Copenhagen, Denmark. ${ }^{3}$ Department of Pathology, Herlev Hospital, University of Copenhagen, Copenhagen, Denmark. ${ }^{4}$ Department of Oncology, Rigshospitalet, University of Copenhagen, Copenhagen, Denmark. ${ }^{5}$ Centre for Genomic Medicine, Rigshospitalet, University of Copenhagen, Copenhagen, Denmark.

Received: 13 December 2017 Accepted: 30 May 2018

Published online: 07 June 2018

\section{References}

1. Carlander A-LF, Grønhøj Larsen C, Jensen DH, Garnæs E, Kiss K, Andersen L, et al. Continuing rise in oropharyngeal cancer in a high HPV prevalence area: a Danish population-based study from 2011 to 2014. Eur J Cancer. 2017:70:75-82.

2. Ferlay J, Soerjomataram I, Dikshit R, Eser S, Mathers C, Rebelo M, et al. Cancer incidence and mortality worldwide: sources, methods and major patterns in GLOBOCAN 2012. Int J Cancer. 2015;136(5):E359-86.

3. Saber CN, Grønhøj Larsen C, Dalianis T, von Buchwald C. Immune cells and prognosis in HPV-associated oropharyngeal squamous cell carcinomas: review of the literature. Oral Oncol. 2016;58:8-13. Elsevier Ltd

4. Grønhøj Larsen C, Jensen DH, Carlander A-LF, Kiss K, Andersen L, Olsen CH, et al. Novel nomograms for survival and progression in HPV+ and HPVoropharyngeal cancer: a population-based study of 1,542 consecutive patients. Oncotarget. 2016;7(44):71761-71772.

5. Garnaes E, Frederiksen K. Kiss K. Andersen L, Therkildsen MH, Franzmann $M B$, et al. Double positivity for HPV DNA/p16 in tonsillar and base of tongue cancer improves prognostication: insights from a large population-based study. Int J Cancer. 2016;139(11):2598-605.

6. Lewis JS. Morphologic diversity in human papillomavirus-related oropharyngeal squamous cell carcinoma: Catch Me If You Can! Mod Pathol. 2017;30:544-53. Nature Publishing Group. Available from: http://www. nature.com/doifinder/10.1038/modpathol.2016.152. [cited 2 June 2017]

7. Lechner M, Frampton GM, Fenton T, Feber A, Palmer G, Jay A, et al. Targeted next-generation sequencing of head and neck squamous cell carcinoma identifies novel genetic alterations in HPV+ and HPV- tumors. Genome Med. 2013:5:49. Available from: http://www.ncbi.nlm.nih.gov/ pubmed/23718828 
8. Zevallos JP, Yim E, Brennan P, Liu AY, Taylor JM, Weissler M, et al. Molecular Profile of Human Papillomavirus Positive Oropharyngeal Squamous Cell Carcinoma Stratified by Smoking Status. Int J Radiat Oncol. 2016;94:864. Elsevier. Available from: http://linkinghub.elsevier.com/retrieve/pii/ S0360301615268605. [cited 14 June 2017]

9. Seiwert TY, Zuo Z, Keck MK, Khattri A, Pedamallu CS, Stricker T, et al. Integrative and comparative genomic analysis of HPV-positive and HPV-negative head and neck squamous cell carcinomas. Clin Cancer Res. 2015;21:632-41. Available from: http://www.ncbi.nlm.nih.gov/pubmed/25056374. [cited 16 June 2017]

10. Lawrence MS, Sougnez C, Lichtenstein L, Cibulskis K, Lander E, Gabriel SB, et al. Comprehensive genomic characterization of head and neck squamous cell carcinomas. Nature. 2015;517:576-82. Nature Publishing Group. Available from: http://www.nature.com/nature/journal/v517/n7536/full/ nature14129.html\#supplementary-information. [cited 28 Jan 2015]

11. Hayes DN, Van Waes C, Seiwert TY. Genetic landscape of human papillomavirus-associated head and neck Cancer and comparison to tobacco-related tumors. J Clin Oncol. 2015;33:3227-34.

12. Saloura V, Vokes EE. EGFR-based bioradiotherapy in SCCHN. Lancet Oncol. 2015;16(2)129-30

13. Argiris A, Heron DE, Smith RP, Kim S, Gibson MK, Lai SY, et al. Induction docetaxel, cisplatin, and cetuximab followed by concurrent radiotherapy, cisplatin, and cetuximab and maintenance cetuximab in patients with locally advanced head and neck cancer. J Clin Oncol. 2010;28:5294-300

14. Burtness B, Bauman JE, Galloway T. Novel targets in HPV-negative head and neck cancer: overcoming resistance to EGFR inhibition. Lancet Oncol. 2013; 14(8):e302-9

15. Garnaes E, Kiss K, Andersen L, Therkildsen MH, Franzmann MB, FiltenborgBarnkob B, et al. Increasing incidence of base of tongue cancers from 2000 to 2010 due to HPV: the largest demographic study of 210 Danish patients. Br J Cancer. 2015;113:131-134. Cancer Research UK. Available from: https:// doi.org/10.1038/bjc.2015.198. [cited 18 June 2015].

16. Garnaes E, Kiss K, Andersen L, Therkildsen MH, Franzmann MB, FiltenborgBarnkob B, et al. A high and increasing HPV prevalence in tonsillar cancers in eastern Denmark, 2000-2010: the largest registry-based study to date. Int J Cancer. 2015;136(9):2196-203.

17. Grønhøj Larsen C, Gyldenløve M, Jensen DH, Therkildsen MH, Kiss K, Norrild B, et al. Correlation between human papillomavirus and p16 overexpression in oropharyngeal tumours: a systematic review. $\mathrm{Br} J$ Cancer. 2014;110:1587-94.

18. Stransky N, Egloff AM, Tward AD, Kostic AD, Cibulskis K, Sivachenko A, et al. The mutational landscape of head and neck squamous cell carcinoma. Science. 2011;333:1157-60.

19. Agrawal N, Frederick MJ, Pickering CR, Bettegowda C, Chang K, Li RJ, et al. Exome sequencing of head and neck squamous cell carcinoma reveals inactivating mutations in NOTCH1. Science. 2011;333:1154-7. Available from: http://www.pubmedcentral.nih.gov/articlerender.fcgi?artid=3162986\&tool= pmcentrez\&rendertype=abstract. [cited 8 Aug 2014]

20. Rubio-Perez C, Tamborero D, Schroeder MP, Antolín AA, Deu-Pons J, PerezLlamas C, et al. In silico prescription of anticancer drugs to cohorts of 28 tumor types reveals targeting opportunities. Cancer Cell. 2015;27:382-96. Elsevier. Available from: http://www.cell.com/article/\$1535610815000574/ fulltext. [cited 9 Mar 2015]

21. Do H, Wong SQ, Li J, Dobrovic A. Reducing sequence artifacts in ampliconbased massively parallel sequencing of formalin-fixed paraffin-embedded DNA by enzymatic depletion of uracil-containing templates. Clin Chem. 2013;59:1376-83.

22. Do H, Dobrovic A. Dramatic reduction of sequence artefacts from DNA isolated from formalin-fixed cancer biopsies by treatment with uracil-DNA glycosylase. Oncotarget. 2012;3:546-58. Available from: http://oncotarget. com/abstract/503

23. 1000 Genomes Project Consortium T 1000 GP, Abecasis GR, Auton A, Brooks LD, DePristo MA, Durbin RM, et al. An integrated map of genetic variation from 1,092 human genomes. Nature. 2012;491:56-65. Available from: http:// www.ncbi.nlm.nih.gov/pubmed/23128226\%5Cnhttp://www.pubmedcentral. nih.gov/articlerender.fcgi?artid=PMC3498066

24. Wong SQ, Li J, Tan AY-C, Vedururu R, Pang J-MB, Do H, et al. Sequence artefacts in a prospective series of formalin-fixed tumours tested for mutations in hotspot regions by massively parallel sequencing. BMC Med Genet. 2014;7:23. Available from: http://bmcmedgenomics.biomedcentral. com/articles/10.1186/1755-8794-7-23. [cited 14 June 2017]
25. Best Practices for Processing HTS Data [Internet]. Available from: http://bestpractices-for-processing-hts-data.readthedocs.io/en/develop_mk/mutect2_ pitfalls.html\%0D.

26. Ramos AH, Lichtenstein L, Gupta M, Lawrence MS, Pugh TJ, Saksena G, et al. Oncotator: Cancer variant annotation tool. Hum Mutat. 2015;36:E2423-9.

27. Mayakonda A, Koeffler HP. Maftools: Efficient analysis, visualization and summarization of MAF files from large-scale cohort based cancer studies. BioRxiv. 2016;52662. Available from: http://www.biorxiv.org/content/early/ 2016/05/11/052662.abstract.

28. Mularoni L, Sabarinathan R, Deu-Pons J, Gonzalez-Perez A, López-Bigas N. OncodriveFML: a general framework to identify coding and noncoding regions with cancer driver mutations. Genome Biol. 2016;17:128. Available from: http://genomebiology.biomedcentral.com/articles/10. 1186/s13059-016-0994-0

29. Alexandrov LB, Nik-Zainal S, Wedge DC, SAJR A, Behjati S, Biankin AV, et al. Signatures of mutational processes in human cancer. Nature. 2013;500:415-21. Available from: http://www.ncbi.nlm.nih.gov/pubmed/23945592. [cited 23 Aug 2017]

30. Burns MB, Temiz NA, Harris RS. Evidence for APOBEC3B mutagenesis in multiple human cancers. Nat Genet. 2013;45:977-83. Available from: http:// www.nature.com/doifinder/10.1038/ng.2701

31. Forbes SA, Bindal N, Bamford S, Cole C, Kok CY, Beare D, et al. COSMIC: mining complete cancer genomes in the Catalogue of Somatic Mutations in Cancer. Nucleic Acids Res. 2011;39:D945-50. Available from: http://www. ncbi.nlm.nih.gov/pubmed/20952405. [cited 6 Sept 2017]

32. Dienstmann R, Jang IS, Bot B, Friend S, Guinney J. Database of genomic biomarkers for cancer drugs and clinical targetability in solid tumors. Cancer Discov. 2015;5:118-23. Available from: http://cancerdiscovery.aacrjournals. org/content/5/2/118. [cited 7 July 2017].

33. Kandoth C, McLellan MD, Vandin F, Ye K, Niu B, Lu C, et al. Mutational landscape and significance across 12 major cancer types. Nature. 2013;502: 333-9. Available from: http://www.nature.com/doifinder/10.1038/nature12634

34. Zhu J, Sammons MA, Donahue G, Dou Z, Vedadi M, Getlik M, et al. Gain-offunction p53 mutants co-opt chromatin pathways to drive cancer growth. Nature. 2015;525:206-11. Available from: http://www.ncbi.nlm.nih.gov/ pubmed/26331536. [cited 2017 Jul 12]

35. Yang SF, Bier-Laning CM, Adams W, Zilliox MJ. Candidate biomarkers for HPV-negative head and neck cancer identified via gene expression barcode analysis. Otolaryngol Neck Surg. 2016;155:416-22. Available from: http:// journals.sagepub.com/doi/10.1177/0194599816642436. [cited 12 July 2017]

36. Wang Z, Wang Y, Ren H, Jin Y, Guo Y. ZNRF3 inhibits the invasion and tumorigenesis in nasopharyngeal carcinoma cells by inactivating the Wnt/ $\beta$ catenin pathway. Oncol Res. 2017;25:571-7. Available from: http://www. ingentaconnect.com/content/10.3727/97818823455816X14760478220149. [cited 12 July 2017]

37. Göke F, Bode M, Franzen A, Kirsten R, Goltz D, Göke A, et al. Fibroblast growth factor receptor 1 amplification is a common event in squamous cell carcinoma of the head and neck. Mod. Pathol. 2013;26:1298-306. Available from: http://www.ncbi.n/m.nih.gov/pubmed/23619603. [cited 12 July 2017]

38. von Mässenhausen A, Franzen A, Heasley L, Perner S. FGFR1 as a novel prognostic and predictive biomarker in squamous cell cancers of the lung and the head and neck area. Ann Transl Med. 2013;1:23. Available from: http://atm.amegroups.com/article/view/2270/3126. [cited 12 July 2017]

39. Koole K, Brunen D, van Kempen PMW, Noorlag R, de Bree R, Lieftink C, et al. FGFR1 is a potential prognostic biomarker and therapeutic target in head and neck squamous cell carcinoma. Clin Cancer Res. 2016; Available from: http://clincancerres.aacrjournals.org/content/early/2016/03/02/1078-0432. CCR-15-1874. [cited 23 Aug 2017]

40. Porta R, Borea R, Coelho A, Khan S, Araújo A, Reclusa P, et al. FGFR a promising druggable target in cancer: Molecular biology and new drugs. Crit Rev Oncol. 2017;113:256-67. Available from: http://www.croh-online. com/article/S1040-8428(17)30085-9/pdf. [cited 23 Aug 2017]

41. Lawrence MS, Sougnez C, Lichtenstein L, Cibulskis K, Lander E, Gabriel SB, et al. Comprehensive genomic characterization of head and neck squamous cell carcinomas. Nature. 2015;517:576-82. Available from: http://www.ncbi. nlm.nih.gov/pubmed/25631445. [cited 6 July 2017]

42. Näsman A, Andersson E, Marklund L, Tertipis N, Hammarstedt-Nordenvall $L$, Attner $P$, et al. HLA class I and II expression in oropharyngeal squamous cell carcinoma in relation to tumor HPV status and clinical outcome. PLoS One. 2013;8:e77025. Available from: http://journals.plos.org/plosone/article?id=10. 1371/journal.pone.0077025 
43. Reinders J, Rozemuller EH, Otten HG, van der Veken LTJN, Slootweg PJ, Tilanus MGJ, et al. HLA and MICA associations with head and neck squamous cell carcinoma. Oral Oncol. 2007;43:232-40.

44. Wichmann G, Herchenhahn C, Boehm A, Mozet C, Hofer M, Fischer M, et al. HLA traits linked to development of head and neck squamous cell carcinoma affect the progression-free survival of patients. Oral Oncol. 2017;69:115-27.

45. McGranahan N, Favero F, de Bruin EC, Birkbak NJ, Szallasi Z, Swanton C. Clonal status of actionable driver events and the timing of mutational processes in cancer evolution. Sci Transl Med. 2015;7:283ra54. Available from: http://www.ncbi.nlm.nih.gov/pubmed/25877892. [cited 7 July 2017]

46. Henderson S, Chakravarthy A, Su X, Boshoff C, Fenton TR. APOBEC-Mediated Cytosine Deamination Links PIK3CA Helical Domain Mutations to Human Papillomavirus-Driven Tumor Development. Cell Rep. 2014;7:1833-41. Available from: http://linkinghub.elsevier.com/retrieve/pii/S2211124714003878.

[cited 7 July 2017]

- fast, convenient online submission

- thorough peer review by experienced researchers in your field

- rapid publication on acceptance

- support for research data, including large and complex data types

- gold Open Access which fosters wider collaboration and increased citations

- maximum visibility for your research: over $100 \mathrm{M}$ website views per year 